\title{
THE OVERABUNDANCE OF MAGNESIUM OVER IRON IN BULGES OF SPIRAL GALAXIES
}

\section{P. JABLONKA}

DAEC,Observatoire de Paris-Meudon, F-92195 Meudon

\author{
N. ARIMOTO
}

Institute of Astronomy, University of Tokyo, Mitaka, Japan

AND

P. MARTIN

Steward Observatory, Tucson, Arizona 85721, USA

We have collected integrated light spectra of bulges of 28 spiral galaxies. Our data sample the Hubble sequence uniformly from S0 to Sd types, and cover a large range in magnitude, viz, about -16 to -22 mag in r-Gunn band. In short, disks do not contribute to more than $14 \%$ of the total integrated light in our spectra, and all galaxies are analyzed under the same conditions. More to read in a forthcoming paper.

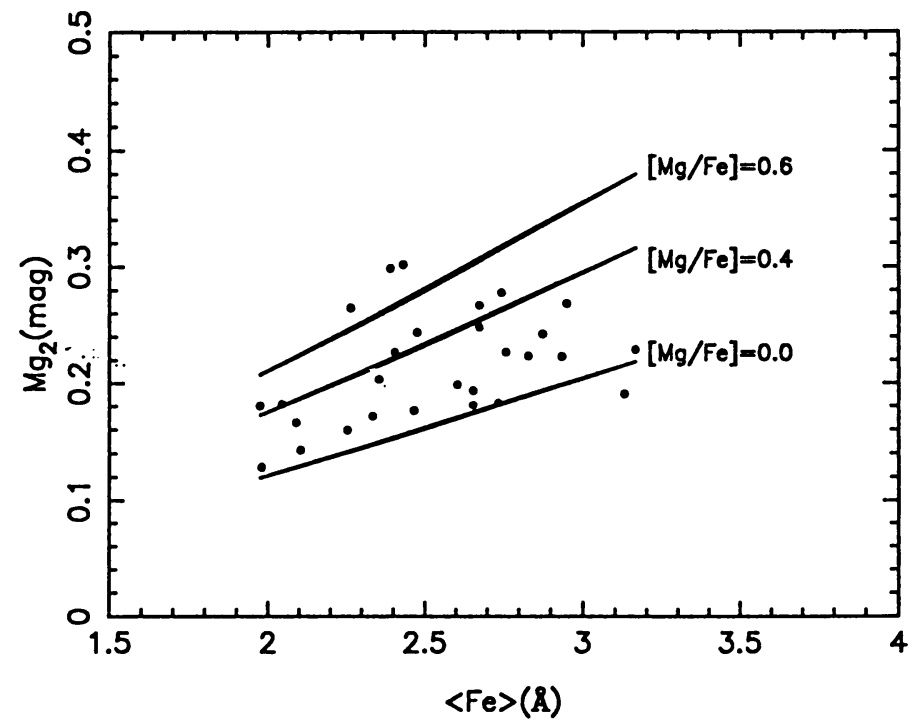

Figure 1. $\mathrm{Mg}_{2}$ vs the mean of the equivalent widths of the iron lines at $5270 \AA$ and $5335 \AA$. The overplotted lines correspond to the predictions of Barbuy et al. model (This volume). 